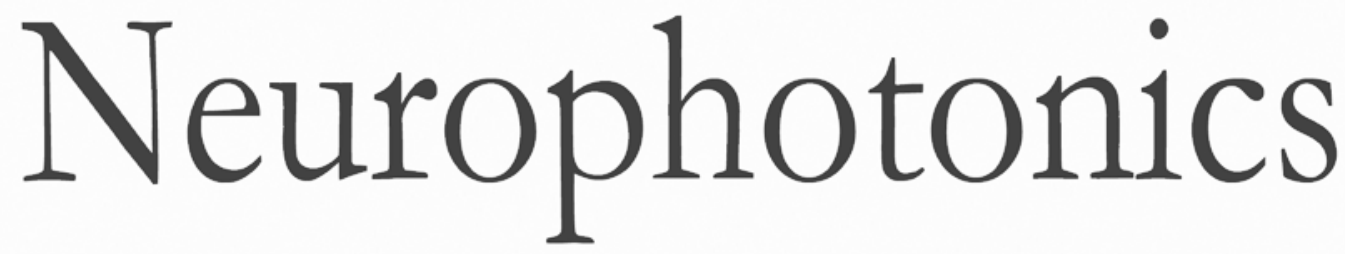

\title{
Cortex-wide multiparametric photoacoustic microscopy based on real-time contour scanning
}

Zhiqiang $\mathrm{Xu}$

Naidi Sun

Rui Cao

Zhengying Li

Quan Liu

Song $\mathrm{Hu}$ 


\title{
Cortex-wide multiparametric photoacoustic microscopy based on real-time contour scanning
}

\author{
Zhiqiang Xu, ${ }^{\mathrm{a}, \mathrm{b}}$ Naidi Sun, ${ }^{\mathrm{b}}$ Rui Cao, ${ }^{\mathrm{b}}$ Zhengying Li, ${ }^{\mathrm{a}}$ Quan Liu, ${ }^{\mathrm{a}}$ and Song $\mathrm{Hu}^{\mathrm{b}, *}$ \\ aWuhan University of Technology, School of Information Engineering, Wuhan, China \\ bUniversity of Virginia, Department of Biomedical Engineering, Charlottesville, Virginia, United States
}

\begin{abstract}
Large-scale, high-resolution imaging of cerebral hemodynamics is essential for brain research. Uniquely capable of comprehensive quantification of cerebral hemodynamics and oxygen metabolism in rodents based on the endogenous hemoglobin contrast, multiparametric photoacoustic microscopy (PAM) is ideally suited for this purpose. However, the out-of-focus issue due to the uneven surface of the rodent brain results in inaccurate PAM measurements and presents a significant challenge to cortex-wide multiparametric recording. We report a large-scale, high-resolution, multiparametric PAM system based on real-time surface contour extraction and scanning, which avoids the prescan and offline calculation of the contour map required by previously reported contour-scanning strategies. The performance of this system has been demonstrated in both phantoms and the live mouse brain through a thinned-skull window. Side-by-side comparison shows that the real-time contour scanning not only improves the quality of structural images by addressing the out-of-focus issue but also ensures accurate measurements of the concentration of hemoglobin $\left(C_{\mathrm{Hb}}\right)$, oxygen saturation of hemoglobin $\left(\mathrm{SO}_{2}\right)$, and cerebral blood flow (CBF) over the entire mouse cortex. Furthermore, quantitative analysis reveals how the out-of-focus issue impairs the measurements of $C_{\mathrm{Hb}}, \mathrm{sO}_{2}$, and $\mathrm{CBF}$. $\odot$ The Authors. Published by SPIE under a Creative Commons Attribution 4.0 Unported License. Distribution or reproduction of this work in whole or in part requires full attribution of the original publication, including its DOI. [DOI: 10.1117/1.NPh.6.3.035012]
\end{abstract}

Keywords: photoacoustic microscopy; contour scanning; concentration of hemoglobin; oxygen saturation of hemoglobin; cerebral blood flow.

Paper 19057R received Jun. 7, 2019; accepted for publication Sep. 4, 2019; published online Sep. 19, 2019.

\section{Introduction}

Providing the unique contrast of optical absorption and excellent scalability of spatial resolution and tissue penetration depth, photoacoustic imaging has emerged as an enabling technology for brain research. ${ }^{1,2}$ Capitalizing on the optical absorption of hemoglobin, multiparametric photoacoustic microscopy (PAM) is uniquely capable of comprehensive characterization of the cerebral vasculature in vivo. ${ }^{3}$ Specifically, statistical, spectroscopic, and correlation analysis of the same dataset allows simultaneous quantification of the total concentration of hemoglobin $\left(C_{\mathrm{Hb}}\right)$, oxygen saturation of hemoglobin $\left(\mathrm{sO}_{2}\right)$, and cerebral blood flow (CBF) at the microscopic level. ${ }^{4}$ Moreover, combing these hemodynamic measurements enables the quantification of the cerebral metabolic rate of oxygen. ${ }^{5}$

In conventional multiparametric PAM, the optical-acoustic dual foci are mechanically translated in the horizontal (i.e., $x-y)$ plane to achieve wide-field volumetric imaging. ${ }^{4}$ Recently, laser scanning has been incorporated to steer the optical beam within the acoustic focus for improved imaging speed. ${ }^{6}$ A problem of the two-dimensional (2-D) raster scan is that the depth of focus of the optical beam is often insufficient to accommodate the depth variation of the mouse brain surface when the entire cortex is imaged. The out-of-focus issue compromises the image resolution and signal-to-noise ratio (SNR). ${ }^{7,8}$

A straight-forward solution is to perform the $z$-stack scanning similar to that in confocal and two-photon microscopy, where sequential 2-D raster scans are performed at different depths to extend the focal zone. ${ }^{9}$ The main drawback of this

*Address all correspondence to Song Hu, E-mail: songhu@virginia.edu approach is that the image acquisition time increases proportionally with the depth range. Recently, three-dimensional (3-D) motorized contour-scanning PAM has been developed to address the out-of-focus issue. ${ }^{4,7}$ In this setting, the scanning process is divided into three sequential steps: (1) perform a 2-D ultrasonic ${ }^{4}$ or photoacoustic ${ }^{7}$ prescan of the region of interest (ROI); (2) extract the 3-D map of the surface contour from the volumetric data acquired by the prescan; and (3) rescan the ROI with the guidance of the 3-D contour map. These additional steps (i.e., prescan and offline calculation of the surface contour map) complicate the process flow and increase the experimental time. In addition, due to the limited focus coverage of the 2-D prescan, the photoacoustic signal in the out-of-focus region (e.g., the periphery of the rodent brain) is typically weak and may even be buried in the background, adding to the challenge of accurately detecting the surface contour.

To overcome these limitations, we have developed a multiparametric PAM implementation, which is capable of extracting the surface contour in real time for large-scale, high-resolution imaging of tissues with uneven surfaces. Different from the previous contour-scanning method that requires the prescan and offline calculation of the contour map, this technique enables online extraction of the surface contour of current cross-sectional scan (i.e., B-scan) to guide the high-resolution acquisition of next B-scan, which leads to a simplified workflow (i.e., one single step rather than three sequential steps) and reduced imaging time. Moreover, the real-time extraction and application of the B-scan contour helps to keep the acquisition of sequential Bscans in focus and maintain the SNR, thereby addressing the poor SNR-induced challenge in contour detection that is associated with the previous contour-scanning method. 
We have tested the performance of the real-time contour scanning-enabled large-scale, high-resolution PAM system in an optically absorbing phantom with curved surface and demonstrated cortex-wide multiparametric imaging of the live mouse brain. Moreover, a side-by-side comparison of the multiparametric brain images acquired by the 2-D raster scan and 3-D real-time contour scan is carried out based on which the influence of the out-of-focus issue on the measurements of $C_{\mathrm{Hb}}, \mathrm{sO}_{2}$, and $\mathrm{CBF}$ is investigated.

\section{Materials and Methods}

\subsection{System Setup}

As shown in Fig. 1(a), the multiparametric PAM system utilizes two nanosecond-pulsed lasers (BX40-2-G, edgewave; wavelength: $532 \mathrm{~nm}$ ). For each laser, the output beam is attenuated by a neutral-density filter (NDC-50C-2M, Thorlabs) and then reduced to an appropriate diameter by an iris (SM1D12D, Thorlabs). After attenuation and reshaping, one of the beams is coupled into a polarization-maintaining single-mode fiber (PM-SMF; F-SPA, Newport) for Raman scattering-based wavelength conversion, ${ }^{10,11}$ which partially red-shifts the 532-nm light. The PM-SMF output is collimated by a collimator (CFC-11X-A, Thorlabs) and purified by a bandpass filter (FB560-10, Thorlabs) to isolate the 558-nm Stokes light. Then, the 558-nm Raman beam and the 532-nm beam from the other optical paths are combined via a dichroic mirror (FF538FDi01, Semrock) and coupled into the imaging head through a two-meter-long regular single-mode fiber (SMF; P1-460B-FC2, Thorlabs). To compensate for the fluctuation in the laser intensity, $5 \%$ of the laser energy is picked off by a beam sampler (BSF10-A, Thorlabs) and monitored by a high-speed photodiode (FDS100, Thorlabs).

In the imaging head, the dual-wavelength beam coming out of the SMF is collimated by an achromatic doublet (AD; AC127-025-A, Thorlabs), reshaped by an iris (SM05D5, Thorlabs), and then focused by a second AD through a correction lens (CL; LA1207-A, Thorlabs) and the central opening of a customized ring-shaped ultrasonic transducer (RT; inner diameter: $2.2 \mathrm{~mm}$; outer diameter: $4.0 \mathrm{~mm}$; focal length: $6.0 \mathrm{~mm}$; center frequency: $35 \mathrm{MHz}$; 6-dB bandwidth: 70\%) onto the object to be imaged. The imaging head is mounted on a three-axis scanner, which consists of two transverse stages (PLS-85, PI miCos $\mathrm{GmbH}$ ) for 2-D raster scan and one vertical

(a)

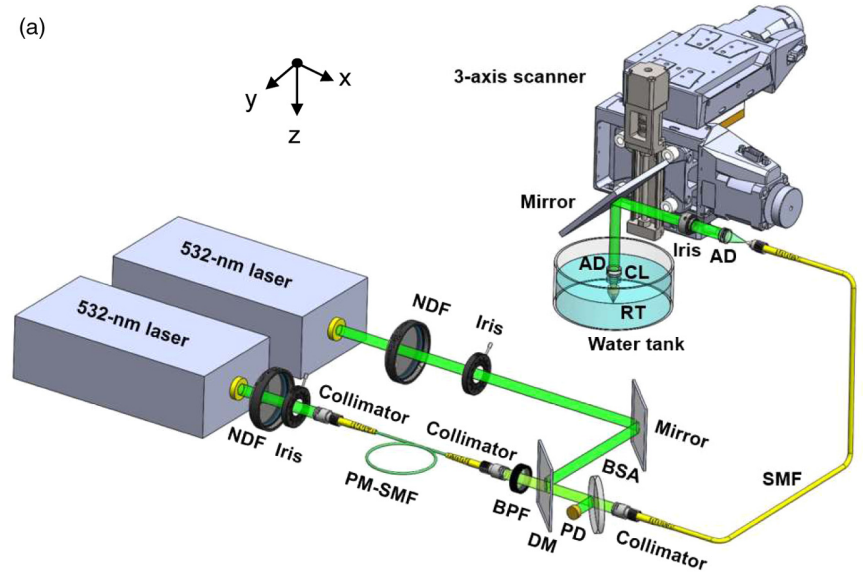

stage (KR15, THK; motor: 28BYG201, Circuit Specialists) for dynamic adjustment of the focal plane. A homemade water tank is used to immerse the RT and CL. A thin layer of ultrasound gel (Aquasonic CLEAR, Parker Laboratories) is applied between the object to be imaged and the transparent polyethylene membrane at the bottom of the water tank for acoustic coupling.

The two lasers, the three-axis scanner, and a data acquisition card (ATS9350, AlazarTech) are synchronized by a field-programmable gate array (PCIe-7841R, National Instruments). The B-scan rate is set to $1 \mathrm{~mm} / \mathrm{s}$, during which the two lasers are alternately triggered to generate dual-wavelength A-line pairs. Statistical, spectroscopic, and correlation analysis of the successive A-line pairs allows simultaneous quantification of $C_{\mathrm{Hb}}, \mathrm{sO}_{2}$, and $\mathrm{CBF}$ at the same spatial scale. ${ }^{12}$

Given the small interval $(10 \mu \mathrm{m})$ of adjacent B-scans, their surface contours are expected to be similar. Therefore, the surface contour of current B-scan can be extracted in real time and used to guide the contour scanning of the next B-scan. The schematic diagram of the real-time contour scanning is shown in Fig. 1(b). The acquisition starts with a conventional B-scan (B-scan \#1) along the $x$-axis without moving the vertical stage. Thus, the trace of the $x-z$ stage appears as a horizontal line, which is denoted as trace 0 . Immediately after B-scan \#1, the acquired data (data \#1) are processed to extract its surface contour, which is denoted as trace 1 . Then, the $y$ stage makes a $10-\mu \mathrm{m}$ step forward and starts B-scan \#2 toward the negative $x$-axis, during which the $z$ stage moves along trace 1 in a reverse fashion. After the completion of B-scan \#2, its surface contour (trace 2) is extracted by combining trace 1 and data \#2. Similarly, B-scan \#3 is acquired by following trace 2 and the surface contour (trace 3 ) is obtained based on trace 2 and data \#3 for the next B-scan. The same procedure is repeated until the completion of the image acquisition.

\subsection{Real-Time Contour Scanning}

As shown in Fig. 2, the z-coordinate of the system's focal plane, which determines the desired position of the imaging head during the contour scan, is input into the software in advance. Once the scan starts, the first B-scan is performed without moving the $z$ stage. Immediately after this B-scan, the coordinate of the curved surface is extracted, based on which the $z$-coordinate to guide the contour scanning of the imaging head in the second $\mathrm{B}$-scan is calculated. Note that a three-step procedure is used to (b)

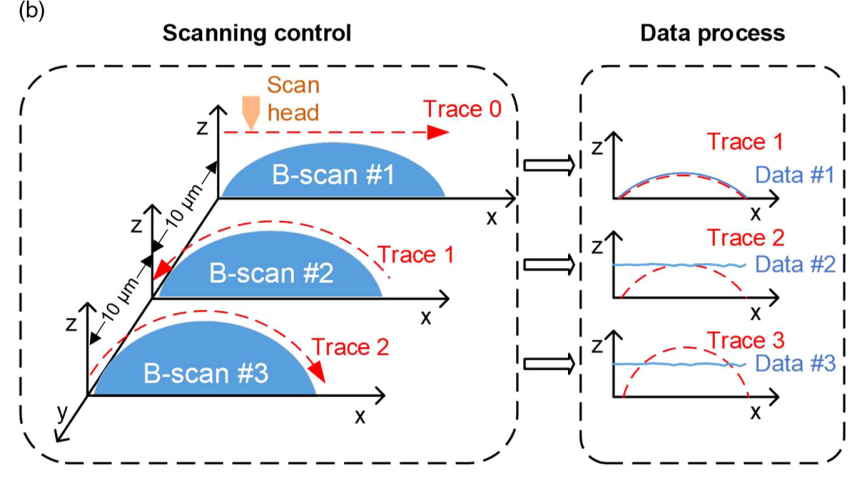

Fig. 1 (a) Schematic of the multiparametric PAM and (b) contour-scan scheme. 


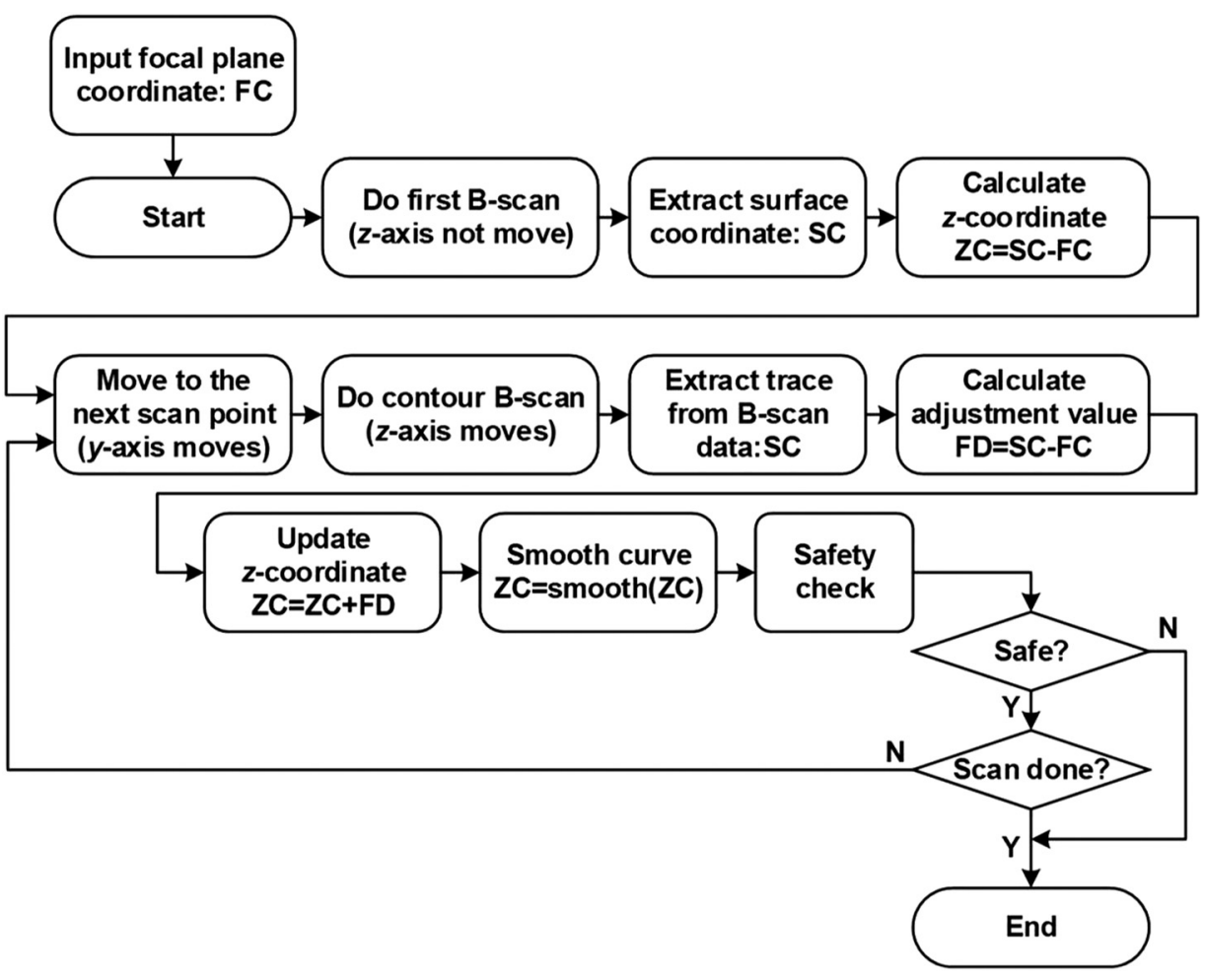

Fig. 2 Flowchart of the algorithm for the real-time contour map extraction. FC, focal plane coordinate; SC, surface coordinate; ZC, $z$-coordinate; FD, feedback data.

extract the $z$-coordinate. First, the maximum signal in each A-line is detected. Then, a curve fitting of these maximum signal points is performed to obtain the surface coordinate of the Bscan. Finally, the $z$-coordinate is calculated by the subtraction of the focal plane coordinate from the surface coordinate.

Contour scanning starts at the second B-scan. Before each contour B-scan, the $y$ stage moves one step $(10 \mu \mathrm{m})$ forward. Then, the imaging head moves along the $x$ and $z$ directions simultaneously to acquire a high-resolution B-scan by following the surface contour extracted from the previous B-scan. After each contour B-scan, the surface coordinate of the B-scan is extracted based on the method mentioned above, which provides feedback on the performance of the contour scanning: the closer the surface coordinate to the focal plane coordinate, the better the performance. If there is a difference between the two coordinates, the $z$-coordinate for next contour B-scan is updated accordingly and then smoothed.

Since this contour scanning strategy is realized without prior knowledge of the whole surface contour map, it is necessary to perform a safety check before starting each contour B-scan. If the upper position, lower position, or the step size exceeds the preset safety range of the $z$ stage, the software terminates the scan and returns an error message. Otherwise, the system starts the next B-scan until the entire image acquisition is completed.

\subsection{Principles of Multiparametric Measurements}

Simultaneous measurement of $C_{\mathrm{Hb}}, \mathrm{sO}_{2}$, and $\mathrm{CBF}$ at the same spatiotemporal scale is realized by statistical, spectroscopic, and correlation analysis of the same PAM dataset. Statistical analysis of the PAM signal allows quantification of the average number of red blood cells (RBCs) within the detection volume as follows:

$$
E\left(N_{\mathrm{RBC}}\right)=\frac{E^{2}\left(A_{\mathrm{PAM}}\right)}{\operatorname{Var}\left(A_{\mathrm{PAM}}\right)-\operatorname{Var}\left(N_{\mathrm{PAM}}\right)},
$$

in which $E$ and Var denote the mean and variance operation, respectively, $A_{\text {PAM }}$ is the amplitude of the PAM signal, and $N_{\text {PAM }}$ is the electronic thermal noise of the PAM system. Since the average amount of hemoglobin in each RBC is 15 pg and the detection volume of our system has been previously quantified to be $263 \mu \mathrm{m}^{3}, C_{\mathrm{Hb}}$ can be calculated as follows: ${ }^{4,5}$

$C_{\mathrm{Hb}}=57 \cdot \frac{E^{2}\left(A_{\mathrm{PAM}}\right)}{\operatorname{Var}\left(A_{\mathrm{PAM}}\right)-\operatorname{Var}\left(N_{\mathrm{PAM}}\right)}(\mathrm{g} / \mathrm{L})$.

Spectroscopic analysis of the dual-wavelength (i.e., 532 and $558 \mathrm{~nm}$ ) A-line pair allows differentiation of oxy- and deoxyhemoglobin $\left(\mathrm{HbO}_{2}\right.$ and $\mathrm{HbR}$, respectively), from which $\mathrm{sO}_{2}$ can be quantified as follows: ${ }^{13}$

$\mathrm{sO}_{2}=\left[\mathrm{HbO}_{2}\right] /\left(\left[\mathrm{HbO}_{2}\right]+[\mathrm{HbR}]\right)$,

in which $\left[\mathrm{HbO}_{2}\right]$ and $[\mathrm{HbR}]$ are the concentrations of $\mathrm{HbO}_{2}$ and $\mathrm{HbR}$, respectively.

Correlation analysis of successive A-lines allows quantification of CBF. Theoretically, the time course of the correlation coefficient follows a Gaussian decay, where the decay constant is linearly proportional to the blood flow speed. ${ }^{14}$ Thus, CBF can be derived by fitting the experimentally measured decorrelation curve with the theoretical model.

\subsection{Animal Preparation}

Male CD-1 mice ( 8 weeks old, Charles River Laboratories) were used for the in vivo experiments. Under general anesthesia, the 
hair in the mouse head was removed and a surgical incision was made in the scalp to expose the skull. Before the PAM experiment, the skull was thinned by a surgical hand drill following removal of the fascia. The skull thinning was performed by following an established protocol to alleviate potential inflammation and avoid possible damage to the brain. ${ }^{15,16}$ Once the desired skull thickness $(\sim 100 \mu \mathrm{m})$ was achieved, the thinnedskull window was cleaned and dried. The ultrasound gel was then applied between the skull window and the ultrasonically and optically transparent polyethylene membrane at the bottom of the water tank. The temperatures of the water tank and the animal body were both maintained at $37^{\circ} \mathrm{C}$. All experimental procedures were carried out in conformity with the animal protocol approved by the Animal Care and Use Committee at the University of Virginia.

\section{Experiments and Results}

\subsection{Phantom Experiments}

An optically absorbing phantom with uneven surface was imaged by the PAM system to demonstrate the feasibility of the real-time contour-scan method. A piece of electrical tape (black) attached to the surface of a plastic ball, the phantom was imaged using the 2-D raster scan and the 3-D contour scan, respectively. Then, the surface contours of the tape in both images were extracted (Fig. 3), in which the $x$-axis represents the B-scan direction and " 0 " in the $z$-axis represents the position of the focal plane (negative and positive values denote that the tape is above and below the focal plane, respectively). As shown in Fig. 3(a), the surface contour of the tape in the raster-scan image is uneven, with most of the region being out-of-focus. In contrast, the surface contour captured by the contour scan is flat, with only a $5-\mu \mathrm{m}$ standard deviation in depth [Fig. 3(b)].

\subsection{In Vivo Experiments}

The side-by-side comparison of raster scan and contour scan was further performed in vivo. Specifically, the entire mouse cortex was first imaged using raster scan to show the influence of the out-of-focus issue on the measurements of microvascular structure and function (i.e., $C_{\mathrm{Hb}}, \mathrm{sO}_{2}$, and $\mathrm{CBF}$ ). Then, the same ROI was reimaged using real-time contour scan to demonstrate the improvement. As shown in Fig. 4(a), the 3-D surface contour of the mouse cortex $(6 \mathrm{~mm} \times 5 \mathrm{~mm})$ extracted from the

(a)

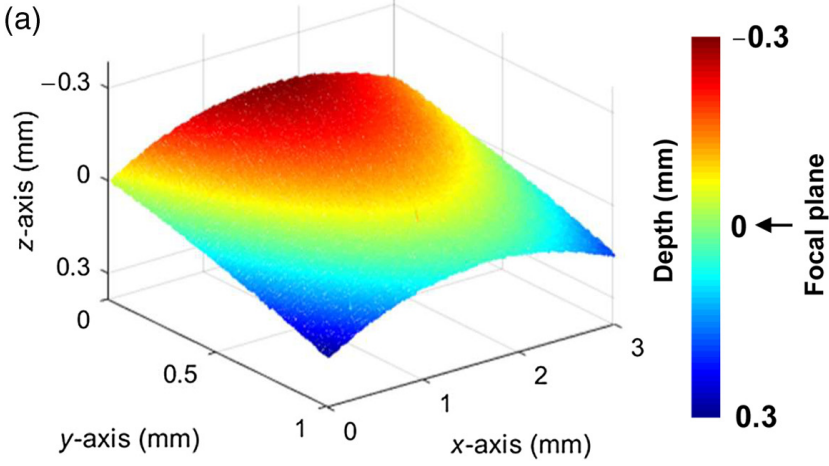

experimental data acquired by raster-scan PAM shows a dome shape with a depth range of $\sim 900 \mu \mathrm{m}$. In contrast, the surface contour of the same cortical ROI imaged by contour-scan PAM is relatively flat, with a $26-\mu \mathrm{m}$ standard deviation in depth. Based on the surface contour map acquired using raster scan, the distance of each vessel away from the focal plane was quantified [Fig. 4(b)], which shows that both the central and peripheral regions of the mouse cortex were out of focus.

Side-by-side comparison of the PAM images acquired using raster scan and contour scan [Figs. 4(c) and 4(d), respectively] clearly shows the adverse influence of the out-of-focus issue on the multiparametric hemodynamic measurements. As indicated by the white arrows, out-of-focus resulted in increased values in the $C_{\mathrm{Hb}}$ and $\mathrm{sO}_{2}$ measurements. The situation in the flow measurement is more complicated, where out-of-focus could lead to either increased or decreased measurement values depending on the distance of the vessel from the focal plane (indicated by the yellow and pink arrows, respectively). Quantitative analysis is provided in Sec. 4 to rationalize these observations.

\section{Discussion}

\subsection{Influence of the Out-of-Focus Issue on Diameter Measurement}

To quantitatively analyze the influence of the out-of-focus issue on the measurement of vessel diameter, side-by-side comparison of the maximum amplitude projection images acquired using raster scan [Fig. 5(a)] and contour scan [Fig. 5(b)] at $532 \mathrm{~nm}$ (an isosbestic point of $\mathrm{HbO}_{2}$ and $\mathrm{HbR}$ ) was performed. Zooming into the periphery of the mouse cortex shows much blurred microvessels in the raster-scan image compared to their counterparts in the contour-scan image [insets in Figs. 5(a) and 5(b)]. This is further evidenced by comparing the cross-sectional profiles of individual vessels in a pair of representative B-scans acquired by the two scanning approaches [Fig. 5(c)]. Clearly, multiple microvascular cross-sections [arrows in Fig. 5(c)] are not resolved due to the compromised spatial resolution in the out-of-focus region. With the aid of segmentation-based single-vessel analysis, ${ }^{3}$ vessel diameter maps of the two structural images were also generated. As highlighted by the arrow pairs, the microvessels in the central and peripheral out-of-focus regions of the raster-scan image [Fig. 5(d)] show larger diameters than their counterparts do in the contour-scan image [Fig. 5(e)]. Furthermore, distribution analysis shows that there are fewer microvessels with less than $30-\mu \mathrm{m}$ diameters in the

Fig. 3 Surface contours of the phantom extracted from the PAM images acquired with (a) raster scan and (b) contour scan.

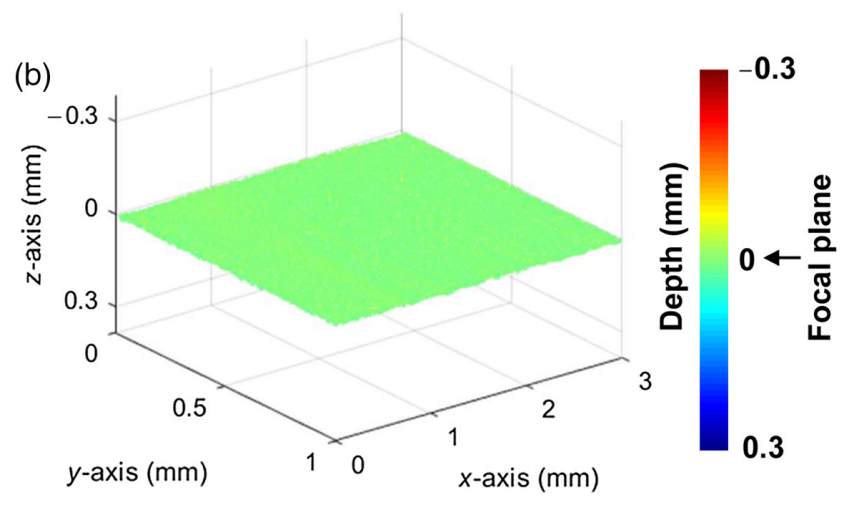


(a)
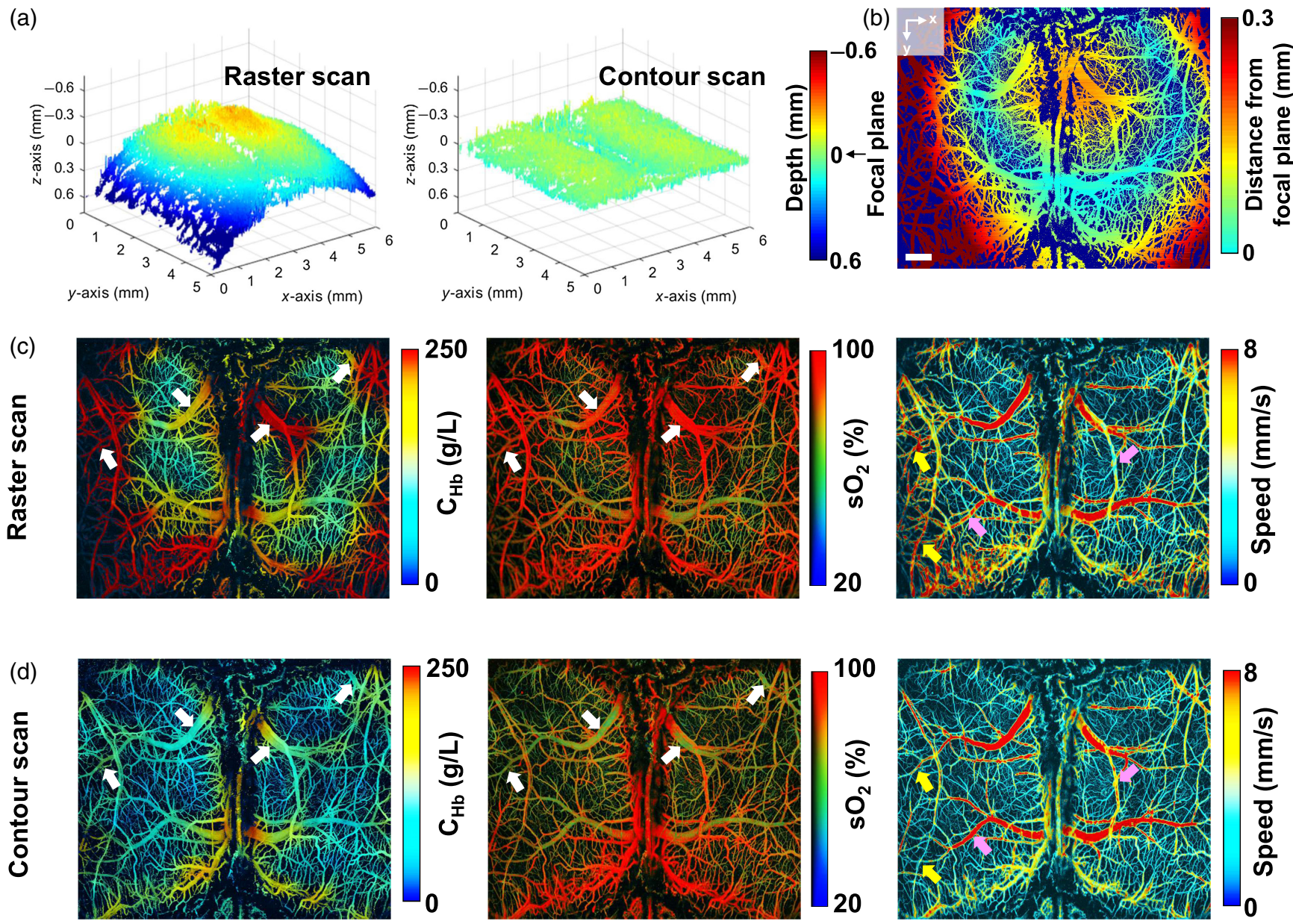

Fig. 4 Side-by-side comparison of raster scan and contour scan in vivo. (a) Surface contours of the entire mouse cortex extracted from the PAM images acquired using raster scan and contour scan, respectively. (b) Pseudocolor map showing the distance of individual vessels from the focal plane in the raster-scan PAM image. (c) Multiparametric PAM images acquired using raster scan. (d) Multiparametric PAM images acquired using contour scan. Scale bar in (b) is $500 \mu \mathrm{m}$ and also applies to (c) and (d). Arrows in (c) and (d) indicate differences between the quantitative measurements by the two types of scanning approaches.

raster-scan image than in the contour-scan image due to the outof-focus issue [Fig. 5(f)]. In conclusion, the structural analysis shows that the real-time contour-scanning PAM enables cortexwide microvascular imaging with high spatial resolution.

\subsection{Influence of the Out-of-Focus Issue on Hemodynamic Measurements}

As shown in Figs. 4(c) and 4(d), the out-of-focus issue leads to increased values in the $C_{\mathrm{Hb}}$ and $\mathrm{sO}_{2}$ measurements. To reveal the underlying mechanism, the $C_{\mathrm{Hb}}$ and $\mathrm{sO}_{2}$ values measured by raster-scan and contour-scan PAM were compared at the singlevessel level. To this end, a four-step procedure was performed: (1) coregister the multiparametric PAM images acquired using raster [Fig. 4(c)] and contour scan [Fig. 4(d)], (2) apply an amplitude threshold to remove the nonvascular background, (3) obtain ratiometric images by taking the ratio of the raster-scan measurements to the contour-scan measurements, and (4) correlate the ratiometric images and the out-of-focus map [Fig. 4(a)] to find out how the $C_{\mathrm{Hb}}$ and $\mathrm{sO}_{2}$ measurements are affected by the degradation in spatial resolution.
According to Eq. (2), $C_{\mathrm{Hb}}$ is codetermined by $E^{2}\left(A_{\mathrm{PAM}}\right)$, $\operatorname{Var}\left(N_{\text {PAM }}\right)$, and $\operatorname{Var}\left(A_{\text {PAM }}\right)$. $\operatorname{Var}\left(N_{\text {PAM }}\right)$ is the electronic thermal noise of the PAM system, which remains the same under both scanning conditions and can be ignored in this analysis. When out of focus, the relaxed point spread function of the optical-acoustic dual foci reduce local optical fluence and compromise acoustic impulse response. Moreover, the averaging effect of the broadened point spread function leads to reduced fluctuation of the PAM signal. Indeed, as shown experimentally in Fig. 6(a), the increased imaging voxel size due to out-of-focus results in reduced $E^{2}\left(A_{\mathrm{PAM}}\right)$ and $\operatorname{Var}\left(A_{\mathrm{PAM}}\right)$. The farther it is away from the focal plane, the larger the decrease. Moreover, $\operatorname{Var}\left(A_{\text {PAM }}\right)$ is more sensitive to the out-of-focus issue than $E^{2}\left(A_{\mathrm{PAM}}\right)$, resulting in increased $C_{\mathrm{Hb}}$. Therefore, the $C_{\mathrm{Hb}}$ value in the raster-scan image is larger than that in the contour-scan image when the vessel is out-of-focus [Fig. 6(b)]. According to Eq. (3), $\mathrm{sO}_{2}$ is codetermined by $\left[\mathrm{HbO}_{2}\right]$ and $[\mathrm{HbR}]$. When out of focus, the photoacoustic amplitudes at both excitation wavelengths (denoted as PA532 and PA558) are reduced. Our analysis shows that PA558 is more sensitive to the out-of-focus issue than PA532 [Fig. 6(c)], resulting in increased $\mathrm{sO}_{2}$. Therefore, 
(a)

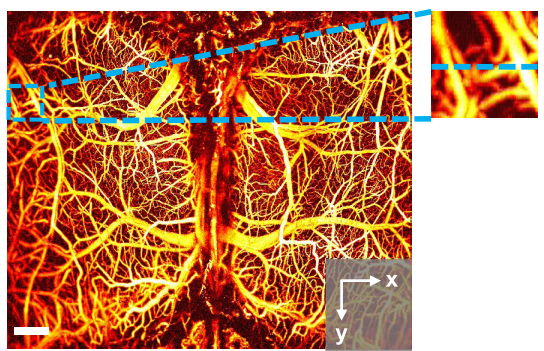

(d)

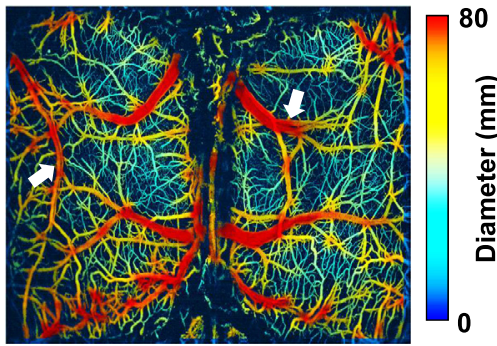

(b)

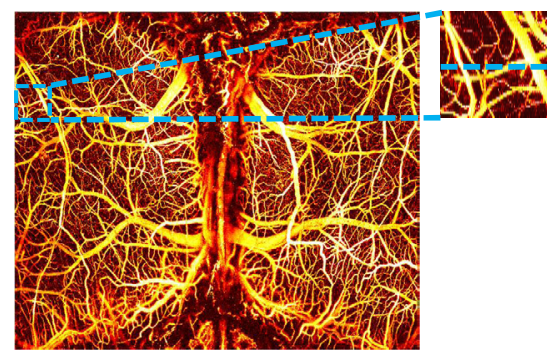

(e)

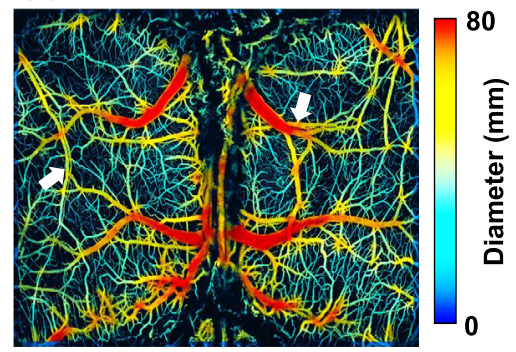

(c)
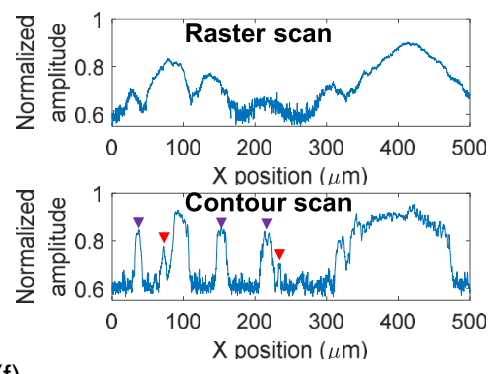

(f) 2.5

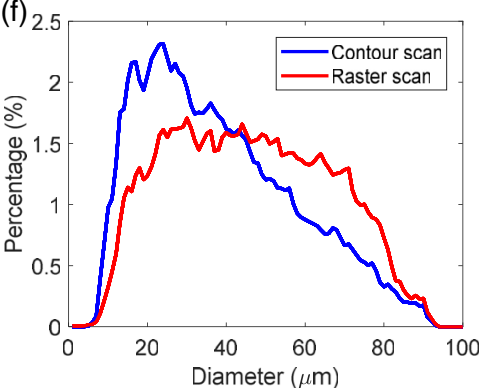

Fig. 5 Influence of the out-of-focus issue on the diameter measurement. (a), (b) Maximum amplitude projection images of the mouse cortex acquired using raster scan and contour scan, respectively. Insets show the blurred microvasculature due to the out-of-focus issue. Scale bar in (a) is $500 \mu \mathrm{m}$ and also applies to (b), (d), and (e). (c) Cross-sectional profiles of the microvessels in the B-scans highlighted by the dashed lines in the insets of (a) and (b). The red arrowheads highlight the vessels that are not resolved in the raster-scan image, and the purple arrowheads highlight vessels that are blurred in the raster-scan image. (d), (e) Vessel diameter maps extracted from the raster-scan and contour-scan images, respectively. (f) Vessel diameter distribution in the raster-scan and contour-scan images. The $x$-axis represents the diameter value and the $y$-axis denotes the ratio of the number of vessel crosssections with a particular diameter to the total number of vessel cross-sections in the image.

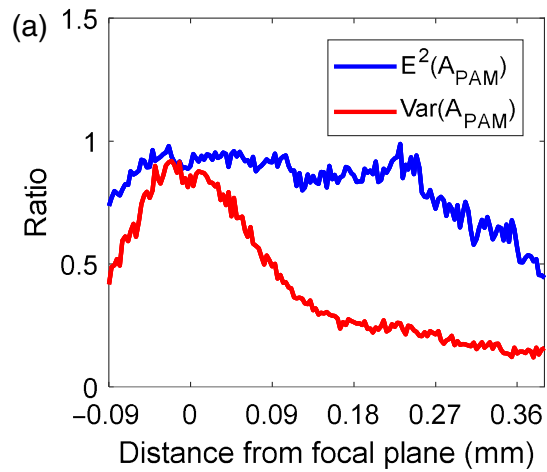

(c)

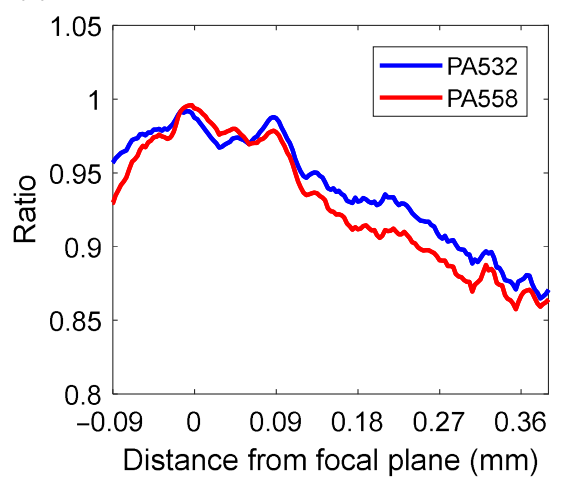

(b)

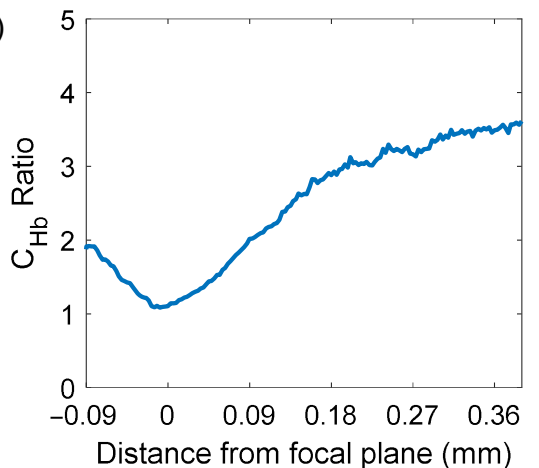

(d)

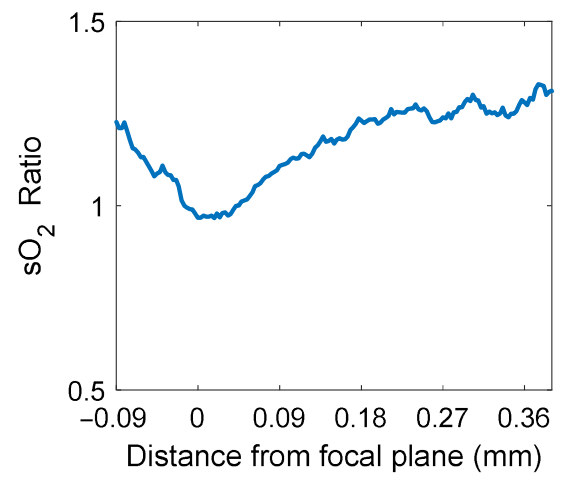

Fig. 6 Influence of the out-of-focus issue on (a) $E^{2}\left(A_{\mathrm{PAM}}\right)$ and $\operatorname{Var}\left(A_{\mathrm{PAM}}\right)$, (b) $C_{\mathrm{Hb}}$, (c) the photoacoustic amplitude (PA532 and PA558, respectively, denote the amplitudes of the photoacoustic signals acquired at 532 and $558 \mathrm{~nm}$ ), and (d) $\mathrm{sO}_{2}$. The ratios are defined as the measurements by raster-scan PAM over those by contour-scan PAM. 
the $\mathrm{sO}_{2}$ value in the raster-scan image is higher than that in the contour-scan image when the vessel is out of focus [Fig. 6(d)].

The side-by-side comparison in Figs. 4(c) and 4(d) shows that out-of-focus also affects the CBF measurement. Difference from the cases of $C_{\mathrm{Hb}}$ and $\mathrm{sO}_{2}$, however, the measured $\mathrm{CBF}$ in the out-of-focus region can be either higher or lower than the true value depending on the distance of the vessel from the focal plane. To unravel the underlying mechanism, we carried out an experiment in a blood vessel-mimicking phantom. As shown in Fig. 7(a), defibrinated bovine blood (Quad Five, 910) was driven by a syringe pump (Pump System Inc., NE-300) to flow through a plastic tube (United States Plastic Corporation, 56514) at $5 \mathrm{~mm} / \mathrm{s}$. The tube was tilted from the focal plane of the PAM system at an angle of $\sim 9 \mathrm{deg}$.

The blood flow within the tube was imaged by both rasterscan and contour-scan PAM for side-by-side comparison [Fig. 7(b)]. In the raster-scan image, the measured flow speed varies with the depth location of the tube along the $y$-axis. In contrast, the contour-scan image shows a relatively constant flow speed of $\sim 5 \mathrm{~mm} / \mathrm{s}$ [Fig. 7(f)], which is consistent with the preset speed. Besides the difference in the measured flow speed, the thickness of the tube in the raster-scan image does not appear uniform, with the two ends thinner than the center, due to the out-of-focus-induced SNR decrease [Fig. 7(c)], which is in contrast to the consistent SNR in the contour-scan image [Fig. 7(e)]. Furthermore, quantitative analysis [Fig. 7(d)] shows that the measured flow speed decreases when the imaged region is slightly out of focus and then starts to increase when it is further out of focus.

This phantom experiment confirms the out-of-focus-induced biphasic changes in the $\mathrm{CBF}$ measurement observed in vivo (Fig. 4) and sheds light on the underlying mechanism. The CBF measurement in multiparametric PAM is based on correlation analysis of the sequentially acquired A-lines, in which the decay constant of the correlation reveals RBC's dwell time in the detection volume. ${ }^{12,17}$ When the imaging area is slightly out of focus, the SNR remains relatively high but the detection volume enlarges, which results in a longer dwell time and thus a lower flow speed. In contrast, when the imaging area is way out of focus, degradation in the SNR becomes non-negligible. As a

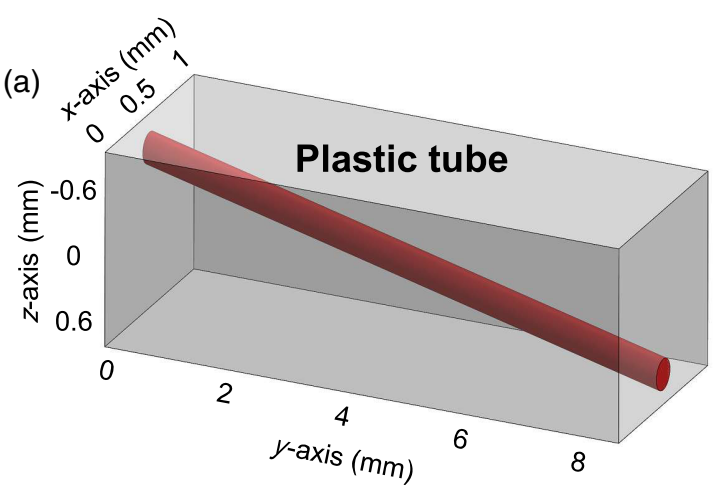

(c)

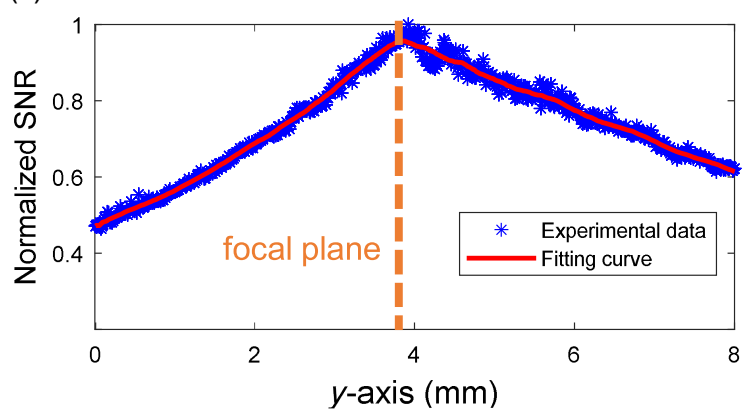

(e)

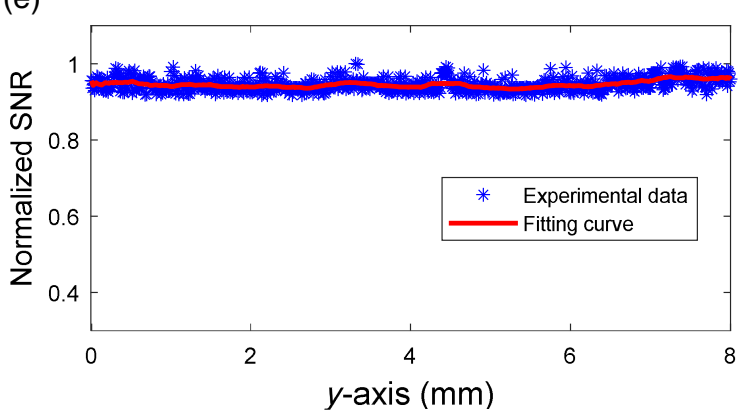

(b)

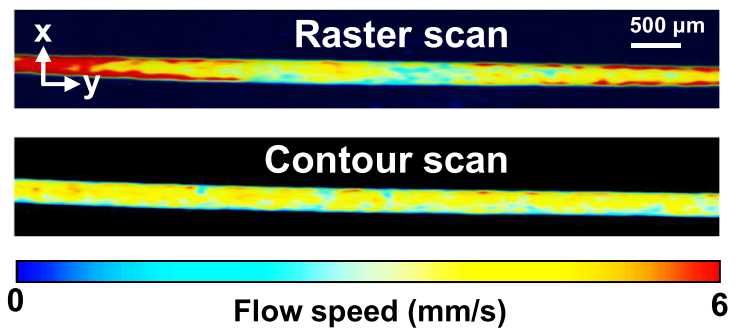

(d)
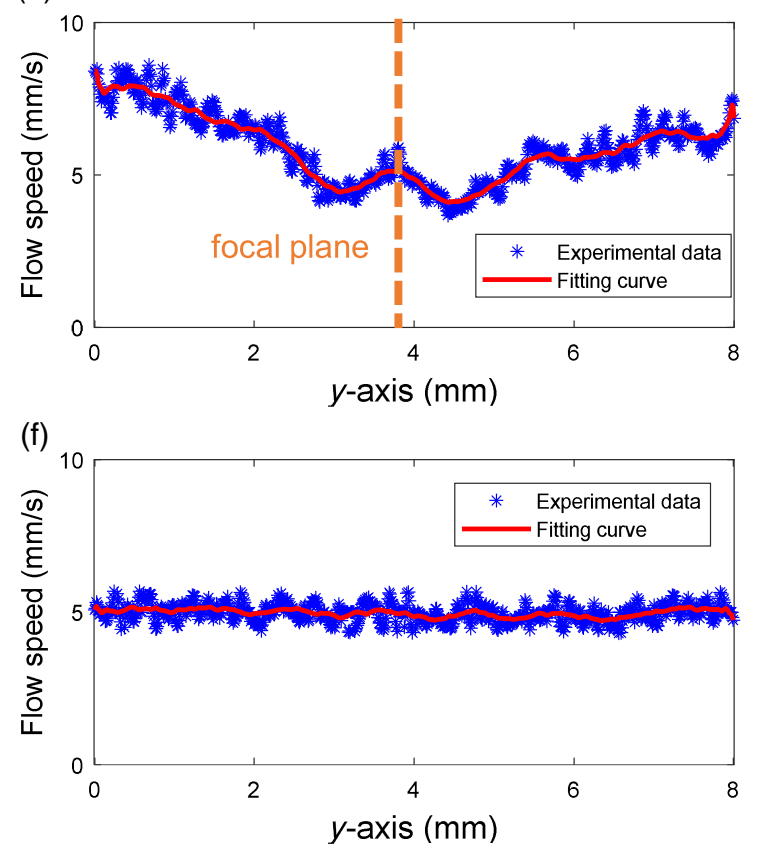

Fig. 7 Phantom experiment showing the influence of the out-of-focus issue on the blood flow measurement. (a) Experimental configuration, (b) measurements of blood flow by raster-scan and contour-scan PAM, respectively. (c), (d) Out-of-focus-induced SNR degradation and flow measurement error in the raster-scan experiment, which are in contrast to the consistent SNR and flow speed measured by the contour scan in (e) and (f). 
result, the rapidly fluctuating noise starts to play a confounding role in the correlation analysis, leading to faster decorrelation of the A-lines and higher flow speed.

\section{Conclusion}

We have developed a real-time, contour-scanning, multiparametric PAM for large-scale, high-resolution imaging of cerebral hemodynamics. The performance of the system has been tested in both the phantom and the live mouse brain. Side-by-side comparison of the real-time contour scan with conventional raster scan shows that this approach can completely eliminate the inaccuracy in the structural and functional measurements induced by the out-of-focus issue in conventional PAM. This technical innovation provides a promising tool for cortex-wide functional brain imaging at the microscopic level.

\section{Disclosures}

No conflicts of interest, financial or otherwise, are declared by the authors.

\section{Acknowledgments}

This work was supported in part by the U.S. National Institutes of Health (Grant No. NS099261), the American Heart Association (Grant No. 15SDG25960005) to S.H., and the Fundamental Research Funds for the Central Universities of China (Grant No. 2016-JL-010) and China Scholarship Council (CSC, Grant No. 201706950020) to Z.X.

\section{References}

1. S. Hu, "Listening to the brain with photoacoustics," IEEE J. Sel. Top. Quantum Electron. 22(3), 117-126 (2016).

2. S. V. Ovsepian et al., "Pushing the boundaries of neuroimaging with optoacoustics," Neuron 96(5), 966-988 (2017).

3. R. Cao et al., "Photoacoustic microscopy of obesity-induced cerebrovascular alterations," Neuroimage 188, 369-379 (2019).

4. B. Ning et al., "Ultrasound-aided multi-parametric photoacoustic microscopy of the mouse brain," Sci. Rep. 5, 18775 (2015).

5. R. Cao et al., "Functional and oxygen-metabolic photoacoustic microscopy of the awake mouse brain," NeuroImage 150, 77-87 (2017).

6. T. Wang et al., "Multi-parametric photoacoustic microscopy of the mouse brain with $300-\mathrm{kHz}$ A-line rate," Neurophotonics 3(4), 045006 (2016).

7. C. Yeh et al., "Microvascular quantification based on contour-scanning photoacoustic microscopy," J. Biomed. Opt. 19(9), 096011 (2014).

8. J. Yang et al., "Synthetic Bessel light needle for extended depth-of-field microscopy," Appl. Phys. Lett. 113, 181104 (2018).

9. C. Zhang, K. Maslov, and L. V. Wang, "Subwavelength-resolution label-free photoacoustic microscopy of optical absorption in vivo," Opt. Lett. 35(19), 3195-3197 (2010).

10. P. Hajireza, A. Forbrich, and R. Zemp, "In-vivo functional opticalresolution photoacoustic microscopy with stimulated Raman scatting fiber-laser source," Biomed. Opt. Express 5(2), 539-546 (2014).
11. P. Hajireza, A. Forbrich, and R. J. Zemp, "Multifocus optical-resolution photoacoustic microscopy using stimulated Raman scattering and chromatic aberration," Opt. Lett. 38(15), 2711-2713 (2013).

12. B. Ning et al., "Simultaneous photoacoustic microscopy of microvascular anatomy, oxygen saturation, and blood flow," Opt. Lett. 40(6), 910-913 (2015)

13. H. F. Zhang et al., "Imaging of hemoglobin oxygen saturation variations in single vessels in vivo using photoacoustic microscopy," Appl. Phys. Lett. 90(5), 053901 (2007).

14. S.-L. Chen et al., "In vivo flow speed measurement of capillaries by photoacoustic correlation spectroscopy," Opt. Lett. 36(20), 40174019 (2011).

15. P. J. Drew et al., "Chronic optical access through a polished and reinforced thinned skull," Nat. Methods 7, 981-984 (2010).

16. G. Yang et al., "Thinned-skull cranial window technique for long-term imaging of the cortex in live mice," Nat. Protocols 5, 201-208 (2010).

17. S.-L. Chen et al., "Photoacoustic correlation spectroscopy and its application to low-speed flow measurement," Opt. Lett. 35(8), 1200-1202 (2010).

Zhiqiang $\mathbf{X u}$ received his BS degree in electronic information engineering from Wuhan University of Technology, Wuhan, China, in 2012. Currently, he is a PhD student in the School of Information Engineering at Wuhan University of Technology and a visiting scholar in the Department of Biomedical Engineering at the University of Virginia. His research focuses on multiparametric photoacoustic microscopy, optical fiber sensing, and high-performance heterogeneous computing.

Naidi Sun received his BS and MS degrees in physical electronics from Harbin Institute of Technology in 2010 and 2012, respectively. Currently, he is a PhD student in the Department of Biomedical Engineering at the University of Virginia. His research focuses on the development of quantitative photoacoustic microscopy and its applications in brain imaging.

Rui Cao received his BS and MS degrees from Nankai University, Tianjin, China, in 2010 and 2013, respectively. He received his PhD from the University of Virginia, Department of Biomedical Engineering, in 2018. His research focuses on multispectral photoacoustic microscopy, molecular photoacoustic imaging, and multimodal optical microscopy.

Zhengying $\mathbf{L i}$ received his $\mathrm{PhD}$ degree from Wuhan University of Technology, China, in 2010. Currently, he is a professor in the School of Information Engineering at Wuhan University of Technology. His research interests include optical fiber sensing and signal processing.

Quan Liu received her $\mathrm{PhD}$ degree from Wuhan University of Technology, China, in 2003. Currently, she is a professor in the School of Information Engineering at Wuhan University of Technology. Her research interests include signal processing, embedded systems, and robots and electronics. In the past five years, she has authored over 60 technical publications, proceedings, editorials, and books.

Song Hu received his BS and MS degrees in electronic engineering from Tsinghua University, Beijing, China, in 2002 and 2005, respectively. He received his $\mathrm{PhD}$ degree in biomedical engineering from Washington University in St. Louis in 2010. Currently, he is an assistant professor of biomedical engineering at the University of Virginia. His research focuses on the development and application of photoacoustic microscopy in brain, cancer, and cardiovascular diseases. 